\title{
REMARKS ON A REMARK OF KAPLANSKY
}

\author{
WILLIAM J. HEINZER AND IRA J. PAPICK
}

(Communicated by Louis J. Ratliff, Jr.)

\begin{abstract}
In his book Commutative rings, Kaplansky makes an interesting remark following the proof of the Hilbert Basis Theorem. He says, "Justly celebrated though this proof is, it leaves one somewhat dissatisfied, since the condition that $I$ and the $I_{n}$ 's be finitely generated is by no means necessary for $J$ to be finitely generated". The purpose of our note is to elaborate on Kaplansky's remark.
\end{abstract}

\section{INTRODUCTION}

Let $R$ be a commutative ring with identity and $J$ an ideal in the polynomial ring $R[x]$. Let $I_{n}$ be the ideal of leading coefficients of polynomials of degree less than or equal to $n$ in $J$ and set $I=\bigcup_{n=0}^{\infty} I_{n}$. In Kaplansky's treatment of the Hilbert Basis Theorem [9, Theorem 69], it is shown that if $I$ and each of the $I_{n}$ 's is finitely generated, then $J$ is finitely generated. Following the proof, Kaplansky makes an interesting remark, which is in effect the primary motivation behind our work. Namely, he says "Justly celebrated though this proof is, it leaves one somewhat dissatisfied since the condition that $I$ and the $I_{n}$ 's be finitely generated is by no means necessary for $J$ to be finitely generated." Our goal is to explore some of the ramifications of this remark. Before describing some of our results, a bit of notation is needed. For the purposes of this note, $R$ will always denote a commutative integral domain with identity, and $R[x]$ the polynomial ring in one indeterminate over $R$. We say $R$ satisfies KP (Kaplansky property) if for each finitely generated ideal $J$ of $R[x]$, the associated ideals $I_{0} \subseteq I_{1} \subseteq I_{2} \subseteq \cdots$ and $I=\bigcup_{n=0}^{\infty} I_{n}$ are all finitely generated, and $R$ satisfies CP (contraction property) if for each finitely generated ideal $J$ of $R[x]$, the associated ideal $I_{0}$ is finitely generated.

$\S 2$ consists of a sequence of lemmas leading up to our result. In particular, we first note (Lemma 2.0) that any domain satisfying CP must be a coherent domain. This sets the stage for the succeeding results. An especially crucial lemma of $\S 2$ (Lemma 2.2) is that if $R$ is a coherent domain and $J$ is a finitely

Received by the editors October 16, 1987. Presented by Ira J. Papick to the special Session in Commutative Algebra at Regional AMS meeting in Lincoln, Nebraska, on October 30, 1987.

1980 Mathematics Subject Classification (1985 Revision). Primary 13F20, 13A15.

The first author's research supported by NSF grant DMS-8521767 
generated ideal in $R[x]$ that contains a monic polynomial, then the ideals $I_{n}$ and $I$ of $R$ associated to $J$ are finitely generated. Our main result states that in the category of Prüfer domains, a domain $R$ satisfies KP if and only if $\operatorname{dim}(R) \leq 1$.

$\S 3$ includes examples, questions and related observations. More specifically, we prove (Example 3.1) that Hochster's example [7, p. 225] of a nontrivial (nonNoetherian, non-Prüfer) 1-dimensional quasilocal coherent domain satisfies KP. It is worth noting that we are unaware of any 1-dimensional quasilocal coherent domain that does not satisfy KP. We also show (Proposition 3.2) that the " $D+$ $M$ construction" does not contribute to the construction of "new" domains satisfying KP.

It follows that integrally closed domains satisfying KP must be completely integrally closed (Proposition 3.3), and hence it seems reasonable to study Krull domains satisfying KP. The class of such domains is strictly larger than the class of integrally closed Noetherian domains, since we show that Eakin and Heinzer's example of a 2-dimensional quasilocal non-Noetherian Krull domain [4, Example 3.1] satisfies KP. We conclude the paper by stating some questions, raising a companion conjecture to a conjecture of Vasconcelos, and providing a few examples in the literal spirit of Kaplansky's remark. In particular, we give an example of a domain $R$, and a fixed finitely generated ideal $J$ in $R[x]$ such that none of the associated ideals of $J$ are finitely generated (Example 3.4). Moreover, we provide an example of a domain $R$, and a fixed invertible ideal $J$ in $R[x]$ such that $J \cap R=I_{0}$ is not a finitely generated ideal of $R$ (Example 3.5).

Any unexplained terminology is standard as in [5 sand 9].

\section{THE PROPERTIES CP AND KP}

In this section we will discuss several different aspects of domains satisfying CP or KP. We shall proceed to our main result through a sequence of lemmas.

Lemma 2.0. If $R$ satisfies $C P$, then $R$ is a coherent domain.

Proof.. Since $R$ is a domain, it suffices to show that the intersection of any two finitely generated ideals $I$ and $J$ is finitely generated [1, I§2, exercise $12 \mathrm{~g}$ ]. This follows easily since $I \cap J=(I x, J(x-1)) \cap R$.

It is interesting to note, that Brumatti, Simis and Vasconcelos have found the equality $I \cap J=(I x, J(x-1)) \cap R$ to be useful in certain computations in computer algebra [2].

Lemma 2.1. If $J$ and $J^{\prime}$ are isomorphic ideals of $R[x]$ with $I_{n}$ and $I$, and $I_{n}^{\prime}$ and $I^{\prime}$ the associated ideals of $R$ to $J$ and $J^{\prime}$ respectively, then the ideals $I_{n}$ and $I$ are finitely generated if and only if the ideals $I_{n}^{\prime}$ and $I^{\prime}$ are finitely generated.

Proof. If $J$ is isomorphic to $J^{\prime}$ as $R[x]$-modules, then there exists polynomials $g(x), g^{\prime}(x) \in R[x]$ such that $J g(x)=J^{\prime} g^{\prime}(x)$. It suffices to consider the case 
where $J=J^{\prime} g^{\prime}(x)$. Assume $\operatorname{deg}\left(g^{\prime}(x)\right)=s$ and the leading coefficient of $g^{\prime}(x)$ is $a \in R$. Thus, for $t \geq s$, we have $I_{t}=a I_{t-s}^{\prime}$. Hence, the ideals $I_{n}$ and $I$ are finitely generated if and only if the ideals $I_{n}^{\prime}$ and $I^{\prime}$ are finitely generated.

The next lemma is especially critical to our main result.

Lemma 2.2. If $R$ is a coherent domain and $J$ is a finitely generated ideal in $R[x]$ that contains a monic polynomial, then the ideals $I_{n}$ and $I$ of $R$ associated to $J$ are finitely generated.

Proof. It suffices to show that the $I_{n}$ 's are finitely generated. Let $R_{n}=R+$ $R x+\cdots+R x^{n}$ and set $A_{n}=J \cap R_{n}$. Note that the image of $A_{n}$ in $R_{n} / R_{n-1}$ is isomorphic as an $R$-module to $I_{n} x^{n}$. Hence, if $A_{n}$ is a finitely generated $R$-module, the $I_{n}$ is a finitely generated ideal of $R$. To see that each $A_{n}$ is finitely generated we first choose $f(x) \in J$ with $f(x)$ monic of degree $m$, and set $R[x] /(f(x))=R[\theta]$. We claim that the $R$-module isomorphism which maps the basis $\left\{1, \theta, \ldots, \theta^{m-1}\right\}$ for $R[\theta]$ to the basis $\left\{1, x, \ldots, x^{m-1}\right\}$ for $R_{m-1}$ restricts to an $R$-module isomorphism of $J /(f(x)) \subseteq R[\theta]$ with $A_{m-1}=$ $J \cap R_{m-1}$. Let $g(x) \in J$ and by the division algorithm $g(x)=f(x) p(x)+q(x)$, where $q(x)=0$ or $\operatorname{deg}(q(x)) \leq m-1$. Note that $q(x) \in J \cap R_{m-1}$, and also the image of $g(x)$ in $J /(f(x))$ equals $q(\theta)$. Hence, the map $q(\theta) \rightarrow q(x)$ is an $R$-module isomorphism of $J /(f(x))$ with $J \cap R_{m-1}=A_{m-1}$.

Finally, since $J$ is a finitely generated ideal in $R[x]$, and $R[\theta]$ is a finitely generated $R$-module, it follows that $J /(f(x))$ is a finitely generated $R$-module. Hence, $J \cap R_{m-1}=A_{m-1}$ is a finitely generated $R$-module, and since $A_{n}=$ $J \cap R_{n}=A_{m-1} \cap R_{n} \subseteq R_{m-1}$ for each $n \leq m-1$, the coherence of $R$ gives that each such $A_{n}$ is also a finitely generated $R$-module [ $1, I \S 2$, Exercise $\left.11 \mathrm{~d}\right]$. Therefore, the $I_{n}$ 's and $I$ are all finitely generated.

Lemma 2.3. If $J$ is an ideal of $R[x]$ of content 1 , and if the only prime ideals of $R$ that contain $J \cap R$ are maximal, then $J$ contains a monic polynomial

Proof. Note that the set $S=\{f(x) \in R[x]: f(x)$ is monic $\}$ is a multiplicatively closed subset of $R[x]$. If $J \cap S=\varnothing$, then there exists a $Q \in \operatorname{Spec}(R[x])$ such that $J \subseteq Q$ and $Q \cap S=\varnothing$. Since $\operatorname{dim}(R / J \cap R)=0$, then $Q \cap R=M$ is a maximal ideal of $R$, and as $c(J)=R$, we get $M[x] \subset Q$. Hence, $Q=(M, f(x))$, where $f(x)$ is a monic polynomial in $R[x]$ such that the image $\bar{f}(x) \in(R / M)[x]$ is irreducible, In particular $Q \cap S \neq \varnothing$, and so we may conclude that $J$ contains a monic polynomial.

One more lemma is needed in preparation for our main result.

Lemma 2.4. If $R$ is a Prüfer domain satisfying $C P$, then $\operatorname{dim}(R) \leq 1$.

Proof. Since CP is preserved under localization, we may assume $R$ is a valuation domain with maximal ideal $M$, and also let us suppose $\operatorname{ht}(M)>1$. Let $P \in \operatorname{Spec}(R)$ such that $P \subset M$ and choose $0 \neq a \in P$ and $b \in M-P$. Note 
that $\bigcup_{n=1}^{\infty}(a): b^{n}=(a, b x-1) R[x] \cap R$, and so CP implies that $\bigcup_{n=1}^{\infty}(a): b^{n}$ is a finitely generated ideal of $R$. (See [2] for a computer algebra application of the equality $(I, b x-1) R[x] \cap R=\bigcup_{n=1}^{\infty} I: b^{n}$, where $b \in R$ and $I$ is an ideal of $R$.) Hence, $(a): b^{t}=(a): b^{t+1}$ for some $t \geq 1$. Let $c=b^{t}$, and note that $c \in M-P$ and $(a): c=(a): c^{2}$. Since $(a) \subseteq(c)$, we have $a=c v$, and it then follows that $(v) \subseteq(c)$ gives $v=c s$. Thus, $(c v): c=(c v): c^{2}$ and so $(v)=(v): c=(c s): c=(s)$. Therefore, $s=v r=c s r$ and $c r=1$, a contradiction.

Theorem 2.5. Let $R$ be a Prüfer domain. Then, $R$ satisfies $K P$ if and only if $\operatorname{dim}(R) \leq 1$.

Proof. By Lemma 2.4, we need only prove that $R$ satisfies KP provided $\operatorname{dim}(R)$ $\leq 1$. We may assume $\operatorname{dim}(R)=1$ and let $J$ be a finitely generated ideal of $R[x]$. First note that an application of the Hilbert syzygies' theorem gives weak $\operatorname{dim}(R[x])=2$, and since $R[x]$ is coherent [8, Théorème (3.4.6), and 11, Proposition 8.2] it follows that $p d_{R[x]} J \leq 1$. We shall consider two cases: (1) $J$ is an invertible ideal of $R[x]$; (2) $p d_{R[x]} J=1$.

(1) Since $R$ is integrally closed, the canonical monomorphism $\operatorname{Pic}(R) \rightarrow$ $\operatorname{Pic}(R[x])$ is an isomorphism [6, Theorem 1.6]. Thus, as $J$ is an invertible ideal of $R[x]$, there exists an invertible fractional ideal $J_{0}$ of $R$ and a nonzero element $q \in q f(R[x])$ such that $J=q J_{0}[x]$. Moreover, we can find a nonzero $r \in R$ such that $r J_{0} \subseteq R$, and hence $r J=r q J_{0}[x]$ gives us that $J \cong r J_{0}[x]$ as $R[x]$-modules. By Lemma 2.1 we see that the ideals associated to $J$ are finitely generated if and only if the ideals associated to $r J_{0}[x]$ are finitely generated. Hence, as $r J_{0}$ is a finitely generated ideal of $R$, this case is complete.

(2) By a result of Vasconcelos [11, Theorem 6.5], we may write $J=L J^{\prime}$, where $L$ is an invertible fractional ideal of $R[x]$ and $\left(R[x]: J^{\prime}\right)=R[x]$. As in case (1), there exists an invertible fractional ideal $L_{0}$ of $R$, a nonzero $u \in q f(R[x])$ with $L=u L_{0}[x]$, and a nonzero $s \in R$ such that $s L_{0} \subseteq R$. Hence, we have the following string of isomorphisms and equalities:

$$
J \cong s J=s L J^{\prime}=s u L_{0}[x] J^{\prime} \cong s L_{0}[x] J^{\prime}=s L_{0} J^{\prime} .
$$

Whence, the ideals associated to $J$ are finitely generated if and only if the ideals associated to $s L_{0} J^{\prime}$ are finitely generated (Lemma 2.1). However, if $I_{n}^{\prime}$ and $I^{\prime}$ are the ideals associated to $J^{\prime}$, then, as $s L_{0}$ is an invertible ideal of $R$, it is straightforward to see that $s L_{0} I_{n}^{\prime}$ and $s L_{0} I^{\prime}$ are the ideals associated to $s L_{0} J^{\prime}$. Therefore, the ideals associated to $J$ are finitely generated if and only if the ideals associated to $J^{\prime}$ are finitely generated. To complete the proof, we will show that $c\left(J^{\prime}\right)=R$ and $J^{\prime} \cap R \neq(0)$, and then by Lemma 2.3, $J^{\prime}$ contains a monic polynomial. Finally, an application of Lemma 2.2 will give that the ideals associated to $J^{\prime}$, and hence $J$, are finitely generated.

Let us assume $c\left(J^{\prime}\right) \neq R$. From the equation $J=L J^{\prime}$, it follows that $J^{\prime}$ is a finitely generated ideal of $R[x]$, and so $c\left(J^{\prime}\right)$ is a finitely generated and 
hence invertible ideal of $R$. Thus, $J^{\prime} \subseteq c\left(J^{\prime}\right)[x] \subset R[x]$. Taking inverses we get

$$
\left(R[x]: c\left(J^{\prime}\right)[x]\right) \subseteq\left(R[x]: J^{\prime}\right)=R[x] .
$$

This is a contradiction, since $c\left(J^{\prime}\right)[x]$ is a nontrivial invertible ideal of $R[x]$. Hence $c\left(J^{\prime}\right)=R$.

Now assume $J^{\prime} \cap R=(0)$. Thus there exists $P \in \operatorname{Spec}(R[x])$ such that $J^{\prime} \subseteq$ $P$ and $P \cap R=(0)$. Moreover, $P$ is an invertible of $R[x]$ [5, Corollary 34.9]. As above, we get $(R[x]: P) \subseteq\left(R[x]: J^{\prime}\right)=R[x]$, which is a contradiction. Therefore, $J^{\prime} \cap R \neq(0)$ and the proof is complete.

\section{EXAMPLES AND OTHER THOUGHTS}

In $\S 2$, we saw (Lemma 2.0 ) that any domain satisfying $\mathrm{CP}$, must be a coherent domain. Lemma 2.4 shows that not all coherent domains satisfy CP. In particular, any Prüfer domain of dimension greater than one does not satisfy CP. However, we do not know of an example of a one dimensional coherent domain that does not satisfy CP.

In [7, p. 225], Hochster provides an example of a one dimensional quasilocal coherent domain that is not a Noetherian domain, and not a valuation domain. We will show that this example satisfies KP. First a lemma is needed.

Lemma 3.0. Let $R \subseteq T$ be an extension of domains with $R$ Noetherian and $T$ a free $R$-module. If $J$ is an ideal of $R[x]$, then the ideals of $T$ associated to $J T[x]$ are finitely generated.

Proof. Write $T=\bigoplus_{\alpha} R t_{\alpha}$, where $\left\{t_{\alpha}\right\}$ is a free module basis for $T$ as an $R$ module. Thus $T[x]=\bigoplus_{\alpha} R[x] t_{\alpha}$ and $J T[x]=\bigoplus_{\alpha} J t_{\alpha}$. Hence each nonzero $f \in J T[x]$ has a unique expression $f=f_{\alpha_{1}} t_{\alpha_{1}}+f_{\alpha_{2}} t_{\alpha_{2}}+\cdots+f_{\alpha}, t_{\alpha}$, where the $f_{\alpha_{i}}$ are nonzero elements of $J$ and $\left\{t_{\alpha_{1}}, \ldots, t_{\alpha_{s}}\right\} \subseteq\left\{t_{\alpha}\right\}$. Also note that $\operatorname{deg}(f)=\max \left\{\operatorname{deg} f_{\alpha_{i}}\right\}$.

Let $I_{n}$ (respectively, $I_{n}^{\prime}$ ) denote the ideal of $R$ (respectively, $T$ ) of leading coefficients of polynomials in $J$ (respectively, $J T[x]$ ) of degree less than or equal to $n$. We claim that $I_{n}^{\prime}=I_{n} T$. If $f \in J T[x]$ and $\operatorname{deg}(f) \leq n$, then from the unique expression $f=f_{\alpha_{1}} t_{\alpha_{1}}+\cdots+t_{\alpha_{s}}, t_{\alpha_{s}}$, we see that the leading coefficient of $f$ in $T$ is in $I_{n} T$. Since the other inclusion is obvious, we get equality. Moreover, as $R$ is Noetherian, $I_{n}$ is a finitely generated ideal of $R$, and so $I_{n} T$ is a finitely generated ideal of $T$. Furthermore, $I=\bigcup_{n=0}^{\infty} I_{n}$ is a finitely generated ideal of $R$, and thus $I=I_{n}$ for some $n$. Therefore, it follows that $I_{n} T$ is the ideal in $T$ of leading coefficients of elements in $J T[x]$.

Example 3.1 [7, p. 225]. The following is an example, due to Hochster, of a nontrivial (non-Noetherian, non-Prüfer) one dimensional quasilocal coherent domain. We will show that this ring satisfies KP. We have altered (up to isomorphism) Hochster's example so that we may directly apply Lemma 3.0 at the appropriate place. 
Let $k$ be a field and $x$ an indeterminate over $k$. Define $R_{n}=k\left[\left[x^{2 / 3^{n}}, x^{3}\right]\right]$, and set $R=\bigcup_{n=0}^{\infty} R_{n}$. Note that each $R_{n}$ is a one dimensional local Noetherian domain, and $R_{m}$ is free over $R_{n}$ for $n \leq m$. Hence $R$ is a nontrivial one dimensional quasilocal coherent domain [1, I§2, exercise $12 \mathrm{e}]$.

We claim that $R$ satisfies KP. Let $J$ be a finitely generated ideal of $R[x]$, and observe that $J$ is extended from $R_{n}[x]$ for some $n$. Since $R[x]$ is a free $R_{n}[x]$-module, it is immediate from Lemma 3.0 that the ideals associated to $J$ are finitely generated.

The method of Example 3.1 is a useful way of producing a variety of nontrivial examples satisfying $\mathrm{CP}$ or KP. Later in this section, we will employ this technique again.

The " $D+M$-construction" is usually a versatile method for constructing a wide variety of examples. However, our next proposition shows that this construction does not contribute to the creation of nontrivial domains satisfying $\mathrm{CP}$ or $\mathrm{KP}$.

Proposition 3.2. Let $V$ be a valuation domain of the form $V=K+M$, where $K$ is a field and $M$ is the maximal ideal of $V$, and let $R=D+M$, where $D$ is a subring of $K$. Then, $R$ satisfies $C P$ if and only if $R$ is Noetherian. In this case, $D$ is a field $[K: D]<\infty$, and $V$ is Noetherian.

Proof. Since one implication is obvious, let us assume $R$ satisfies CP. We first claim that $D$ is a field. Suppose not and let $(0) \neq P \in \operatorname{Spec}(D)$. Choose $0 \neq a \in M$ and $b \in(P+M) \backslash M$. Proceeding as in the Proof of Lemma 2.4, CP implies that $\bigcup_{n=1}^{\infty}(a): b^{n}$ is a finitely generated ideal of $R$, and thus $(a): c=(a): c^{2}$ for some $c \in(P+M) \backslash M$. Note that $a V \subseteq c V \quad(=V)$, and so $a=c v$ for some $v \in V$. Since $a \in M$ and $c \notin M$, we have $v \in M \subseteq R$. Thus, $v V \subseteq c V \quad(=V)$, which gives $v=c s$ for some $s \in V$, and hence $s \in M \subseteq R$. From this point on the proof is identical to Lemma 2.4 and we reach the desired contradiction. Therefore $D=k$ is a field.

By Lemma 2.0, we know that $R$ is coherent, and so $[K: k]<\infty$ and $M$ is a finitely generated ideal of $R$ [3, Theorem 3]. The proof will be complete by showing $\operatorname{dim}(R)=1$. This follows in the same manner as above with the aid of the fact that $\operatorname{Spec}(R)=\operatorname{Spec}(V)$.

Thus far we have seen that the class of domains satisfying CP or KP enjoys some nice structural features. Our next proposition adds to this list.

Proposition 3.3. Let $R$ be an integrally closed domain satisfying $C P$ and let $P(R)=\{P \in \operatorname{Spec}(R): P$ is minimal over $((a): b)$ for some $a, b \in R\}$. Then $P(R)=\{P \in \operatorname{Spec}(R): \operatorname{ht}(P)=1\}$ and $R=\bigcap_{P \in P(R)} R_{P}$, where each $R_{P}$ is a rank one valuation domain. In particular, $R$ is completely integrally closed.

Proof. Clearly each height one prime ideal of $R$ is in $P(R)$. Let $P \in P(R)$, and note that the hypotheses localize. We claim that $R_{P}$ is a valuation domain. Let $J$ be a finitely generated ideal of $R_{P}$. Since $R_{P}$ satisfies $\mathrm{CP}$, and thus is 
coherent (Lemma 2.0), we see that $J^{-1}=\left(R_{P}: J\right)$ is a finitely generated $R_{P^{-}}$ module. Hence $\left(J J^{-1}\right)^{-1}=R_{P}$, as $R_{P}$ is integrally closed [9, exercise 39(a), p. 45]. Therefore, $J J^{-1} \nsubseteq P R_{P}\left[10\right.$, Theorem E], and so $J J^{-1}=R_{P}$, which implies that $R_{P}$ is a one dimensional valuation domain (Lemma 2.4). It follows in general that $R=\bigcap_{P \in P(R)} R_{P}$ [10, Theorem E], and in this particular case we may conclude that $R$ is completely integrally closed.

In view of Proposition 3.3, it is natural to determine which Krull domains satisfy CP or KP. One immediate point that can be made is that not all such domains are Noetherian. In particular, Eakin and Heinzer have given an example of a two-dimensional quasilocal non-Noetherian Krull domain [4, Example 3.1], and by arguing as in Example 3.1, it is straightforward to see that their example satisfies KP.

Another area of interest revolves around the following conjecture of Vasconcelos: If $R$ is a one-dimensional coherent domain, then the integral closure of $R$ is a Prüfer domain. An analogous conjecture is the following: If $R$ is a one-dimensional domain satisfying CP or KP, then the integral closure of $R$ is a Prüfer domain. Of course, this could be identical to the Vasconcelos conjecture, since, as mentioned before, we have no example of a one-dimensional coherent domain that does not satisfy KP.

We conclude this paper with some final comments and a few examples in the spirit of Kaplansky's original remark. Note that by Lemma 2.4 and Theorem 2.5 , the properties $\mathrm{CP}$ and $\mathrm{KP}$ are equivalent in the class of Prüfer domains. For which other classes of domains are the properties CP and KP equivalent? Certainly, if we do not quantify over all finitely generated ideals $J$ of $R[x]$, it is easy to construct examples where $I_{0}$ is finitely generated, but $I_{1}$ is not. More generally, for a fixed finitely generated ideal $J$ of $R[x]$, it can happen that $I_{0}, I_{1}, \ldots, I_{n-1}$ are all finitely generated ideals of $R$, but $I_{n}$ is not. For if, say $J^{\prime}$ is a finitely generated ideal of $R[x]$ such that $J^{\prime} \cap R$ is not finitely generated, then for $J=x^{n} J^{\prime}$ we have $I_{0}=\cdots=I_{n-1}=(0)$ and $I_{n}=J^{\prime} \cap R$.

Recall that Kaplansky's remark emphasized that given a finitely ideal $J$ in $R[x]$, one should not expect that the $I_{n}$ 's or $I$ be finitely generated in $R$. Our next example shows this in the extreme. In particular, we give an example of a domain $R$, and a finitely generated ideal $J$ in $R[x]$, such that none of the associated ideals of $J$ are finitely generated.

Example 3.4. Let $K$ be a field, and let $A$ be the polynomial ring $A=$ $K[t, y, z]$. Let $M=(y, z) A$ and set $R=K+M$, and $P=y A \cap R$. Note that $P=(y):{ }_{R} z=\bigcup_{n=1}^{\infty}(y): z^{n}$, and in terms of irredundant generators, $P=\left(y, t y, t^{2} y, \ldots\right)$. Hence, $P$ is not finitely generated as an ideal of $R$, and moreover, the ideal $(P, z)$ in $R$ is not finitely generated. Let $J$ be the finitely generated ideal of $R[x], J=(y, z x-1) R[x]$. Observe that $I_{0}=P$ (see the proof of Lemma 2.4), and $I_{1}=I_{2}=\cdots=I=(P, z)$. Therefore, all the associated ideals of $J$ are not finitely generated. 
The above example displays in a strong way that the associated ideals of a finitely generated ideal $J$ of $R[x]$ need not be finitely generated. What about the associated ideals of an invertible ideal $J$ of $R[x]$ ? If $R$ is seminormal, then the canonical monomorphism $\operatorname{Pic}(R) \rightarrow \operatorname{Pic}(R[x])$ is an isomorphism [6, Theorem 1.6], and so any invertible $J$ of $R[x]$ is isomorphic to an extended ideal $L[x]$, where $L$ is an invertible ideal of $R$. Hence, as the ideals associated to $L[x]$ are obviously finitely generated, it follows from Lemma 2.1 that the ideals associated to $J$ are finitely generated. If $R$ is not seminormal, then it need not be the case that the ideals associated to an invertible ideal are finitely generated. This is precisely the situation in our next example. In particular, we give an example of a domain $R$, and an invertible ideal $J$ in $R[x]$, such that $J \cap R=I_{0}$ is not finitely generated.

Example 3.5. Let $K$ be a field of characteristic 2, and let $V$ be a rank one nondiscrete valuation domain of the form $K+M$, where $M$ is the maximal ideal of $V$. Let $0 \neq a \in M$ and set $R=K+a M$. We claim that $J=$ $\left(a^{2}+a^{3} x, a^{4}\right)$ is an invertible ideal of the polynomial ring $R[x]$, and $J \cap R$ is not a finitely generated ideal of $R$. To see this, first note that $J^{2}=\left(a^{4}+\right.$ $\left.a^{6} x^{2}, a^{8}\right)=a^{4} R[x]$. As one inclusion is obvious, let us demonstrate the other by showing that $a^{4} \in J^{2}$. This follows, since $a^{2}\left(a^{4}+a^{6} x^{2}\right) \in J^{2}$ and $a^{8} \in J^{2}$ gives $a^{6} \in J^{2}$, and thus $a^{4}+a^{6} x^{2} \in J^{2}$. Therefore, $J^{2}=a^{4} R[x]$, and so $J$ is an invertible ideal in $R[x]$.

We will now prove that $J \cap R=a^{3} M$, and hence $J \cap R$ is not a finitely generated ideal of $R$. First we verify that $a^{3} M \subseteq J \cap R$. Let $b \in M$ and consider the calculation, $\left(a^{2}+a^{3} x\right)\left(a b+a^{2} b x\right)=a^{3} b+a^{5} b x^{2}$. Since $a^{5} b x^{2} \in a^{4} R[x]$, it follows that $a^{3} b \in J \cap R$. For the reverse inclusion, let $c \in J \cap R$. Then $c=$ $f(x)\left(a^{2}+a^{3} x\right)+a^{4} g(x)$, where $f(x), g(x) \in R[x]$. Write $f(x)=r_{0}+r_{1} x+$ $\cdots+r_{n} x^{n}$ and $g(x)=s_{0}+s_{1} x+\cdots+s_{m} x^{m}$. Since $c \in R$, we have the coefficient of $x$ in the expression of $c$ must be 0 , i.e., $r_{1} a^{2}+r_{0} a^{3}+s_{1} a^{4}=0$. Thus, $r_{1}+r_{0} a+s_{1} a^{2}=0$. Write $r_{1}=u+a d$, where $u \in K$ and $d \in M$. Hence, $u=-\left(a d+r_{0} a+s_{1} a^{2}\right)=0$, and moreover, $d+r_{0}+s_{1} a=0$. Write $r_{0}=v+a e$, where $v \in K$ and $e \in M$, and notice that $v=-\left(a e+d+s_{1} a\right) \in K \cap M=(0)$. Thus, $r_{0}=a e$, and so $c=r_{0} a^{2}+a^{4} s_{0} \in a^{3} M$. Therefore, $J \cap R=a^{3} M$, and hence $J \cap R$ is not a finitely generated ideal of $R$.

\section{ACKNOWLEDGMENT}

We are thankful to Wolmer V. Vasconcelos for some enlightening discussions pertaining to this paper.

\section{REFERENCES}

1. N. Bourbaki, Commutative algebra, Addison-Wesley, Reading, Mass., 1972.

2. P. Brumatti, A. Simis and W. V. Vasconcelos, Normal Rees algebras, J. Algebra (to appear). 
3. D. E. Dobbs and I. J. Papick, When is $D+M$ coherent?, Proc. Amer. Math. Soc. 56 (1976), 51-54.

4. P. Eakin and W. Heinzer, Non finiteness in finite dimensional Krull domains, J. Algebra 14 (1970), 333-340.

5. R. Gilmer, Multiplicative ideal theory, Dekker, New York, 1972.

6. R. Gilmer and R. C. Heitmann, On Pic $(R[x])$ for $R$ seminormal, J. Pure Appl. Algebra 16 (1980), 251-257.

7. S. Glaz and W. V. Vasconcelos, Flat ideals. III, Comm. Algebra 12 (1984), 199-227.

8. L. Gruson and M. Raynaud, Critères de platitude et de projectivité, Invent. Math. 13 (1971), $1-89$.

9. I. Kaplansky, Commutative rings, Allyn and Bacon, Boston, Mass., 1970.

10. H. Tang, Gauss' Lemma, Proc. Amer. Math. Soc. 35 (1972), 372-376.

11. W. V. Vasconcelos, The rings of dimension two, Lecture Notes in Pure and Appl. Math., vol. 22, Dekker, New York, 1976.

Department of Mathematics, Purdue University, West Lafayette, Indiana 47907

Department of Mathematics, University of Missouri, Columbia, Missouri 65211 